\title{
Ablation-assisted current interruption in medium voltage switchgear - Development and prospect
}

\author{
Henning Taxt \\ Norwegian University of Technology and Science
}

\begin{abstract}
$\mathrm{SF}_{6}$-gas is widely used in medium and high voltage switchgear today because of its excellent insulation and current interruption capabilities. However, due to its high global warming potential, there is a political pressure to replace it wherever possible, resulting in a need for new solutions in the design of compact lowcost switchgear. An evident response is to review and further develop the technologies that were promising before the entry of $\mathrm{SF}_{6}$. One of these was the application of gas-emitting insulation materials, in a process of ablation.

This paper reviews the range of commercial breaker designs that have taken advantage of ablation in the interruption process from the 1930s until today. The main designs and developments are outlined and some important parameters are explained, such as the steady state ablation-dominated arc, arc quenching and dielectric recovery in the presence of ablation material.

The ratings of the mentioned products, as well as more recent experiments, indicate that there is a design limit for simple ablation-assisted breakers at around $20 \mathrm{kV}$, but the reasons for the apparent limit are not thoroughly studied or explained.

Reviving the knowledge of ablation breakers, and combining this with new knowledge and new tools could prove valuable to the development of $\mathrm{SF}_{6}$-free MV switchgear.
\end{abstract}

\section{Introduction}

From the early applications of electricity, there has been a need to interrupt safely any current that may occur in the electric system. The requirements to breakers have varied through time, depending on application and available technology. Today a large range of products is available, answering to different needs. The present paper focuses on switching technology for medium voltage $(1-52 \mathrm{kV})$, and especially the interruption of load currents (up to $1250 \mathrm{~A}$ ).

In order to reduce their size, a large share of metalenclosed switchgear at medium and high voltage contain $\mathrm{SF}_{6}$-gas, an excellent gas for insulation and current interruption. There is a political pressure to reduce and finally eliminate the use of $\mathrm{SF}_{6}$ in electrical equipment, due to its extremely high global warming potential (GWP) and long atmospheric lifetime. With the increased knowledge and awareness of global warming, the application of $\mathrm{SF}_{6}$ has become an issue of regulation, and further regulatory measures to limit its use in electrical equipment are expected. This is the main driver for further development of breakers. One of today's great challenges is to retrofit $\mathrm{SF}_{6}$-free switchgear into the very compact designs that have developed, without reducing voltage and current ratings. Several existing products, mostly of pre-SF 6 origin, apply ablation as an active element in the current interruption. Briefly explained, ablation is the process of removing a thin layer of material. The intense heat of the electric arc evaporates a thin layer of insulating material. This evaporation process, and the resulting gas, cools the arc, causes a reduction in arc conductivity and improves the arc-quenching properties. Although products have existed for decades, little information is publicly available on the performance and limitations of these designs. In order to improve the technology, it is fruitful to review the relevant designs and available research on the topic. For example, ablation is important and well known from current interruption in low voltage moulded-case circuit breakers (MCCB).

In this paper, I present the state-of-the-art knowledge on ablation-assisted interruption, limited to that relevant for the development of medium voltage switchgear. I start by explaining the basics of current interruption and breaker technologies, before I review the existing medium voltage products that actively apply ablation in arc quenching. In the following section, I will present the most relevant physical interpretations of ablationdominated arcs and interruption of these. More specifically, as publications focusing on medium voltage interruption are scarce, I present elements from research on low and high voltage breakers that are relevant for the general understanding of ablationassisted interruption. The main topics are the effect of ablation in the high current region, the current-zero region (thermal interruption) and the post-arc region (dielectric recovery).

\section{Background}

\subsection{Ac current interruption}

In most ac power-switching equipment the interruption follows these basic steps: The contacts are separated and an electric arc ignites in the contact gap. When the current reaches its current zero, the current is interrupted. For this interruption to take place, the electric conductivity of the medium between the contacts must be sufficiently reduced to stop the current from flowing in the opposite direction after current zero (arc quenching). In addition, the interrupting medium must regain sufficient dielectric strength to avoid 
breakdown and re-ignition of the electric arc, as the breaker must sustain the total voltage of the interrupted circuit (recovery). Both arc quenching and recovery must be successful to ensure a successful interruption.

\subsection{Breaker technologies for medium voltage} Oil, air, $\mathrm{SF}_{6}$ and vacuum are the most common media for arc interruption.

Oil is a good interruption medium because it is insulating in its normal condition and during interruption, the arc causes the oil to decompose to hydrogen, which has a very high thermal conductivity, efficiently cooling the electric arc. Once the arc is quenched, the gas bubble collapses and the contact gap is filled by oil. Explosion hazard is a serious drawback for oil-filled breakers.

Interruption in vacuum is prevalent in new medium voltage circuit breakers, but these are expensive compared to standard load-break switches, which have significantly lower current rating. Vacuum interruption will not be treated further in this paper, as the physical processes in play are quite different.

Gas-blast circuit breaker operates by providing a flow of gas onto the electric arc, which cools and quenches the arc. Air has traditionally been the interruption medium, and a pressure tank or a puffer mechanism, explained in section 3.3, can provide the air-blast. One of the most significant shifts in the development of breakers was the utilization of $\mathrm{SF}_{6}$ as the interruption medium. The gas has the combination of high dielectric strength and good current interrupting capabilities that makes it extraordinarily well suited for use in medium and high voltage switchgear. In the last decades, it has allowed the development of cheaper and more compact equipment.

One advantage of applying ablation is that no pressure tank or mechanical force is needed, except for contact movement. Another is that the effect of ablation is selfadjusting, as an increased current will lead to a higher rate of ablation. The ablation breaker can also have a current limiting effect because of its high arc voltage compared to e.g. air blast or vacuum breakers. This effect is negligible on higher voltage levels.

There are also some obvious challenges with applying ablation in load break switches. A small amount of ablation material is consumed in every current interruption, and the number of operations are therefore limited. The exact number depends on design. Soot contamination from ablation inside the metal enclosure is another challenge that must be dealt with.

\section{Existing designs for MV ablation- assisted interruption}

\subsection{Early application of ablation-assisted switching}

An early design for ablation in medium voltage switches is presented in Figure 1. The development of this medium voltage load break switch arose from the discovery that the insulating nozzle of an air-blast breaker could improve the interruption capabilities of the breaker, even without applying the air blast. As an explanation, Mayr suggests that the arc evaporates a thin layer of nozzle material that pushes the arc away from the nozzle surface and cools it [1]. The paper treats in particular the challenge of material wear with different current amplitudes. Due to contact and material wear, the number of operations is limited to 300 at $100 \mathrm{~A}$, and only three operations at $1000 \mathrm{~A}$.

An improved design presented in 1938 increase the voltage rating to $20 \mathrm{kV}$, nominal current to $400 \mathrm{~A}$ and short circuit interruption capability to 200 MVA [2]. The improvement, referring to Figure 2, consists mainly in replacing the contact pin by a contact tube (8), and introducing an ablation pin (6) fitted into the mentioned contact tube. This resulted in a narrow annular gap for arc movement between the ablation pin and the outer ablation tube (4 and 5), increasing the level of arc-wall interaction and the surface-to-volume ratio.

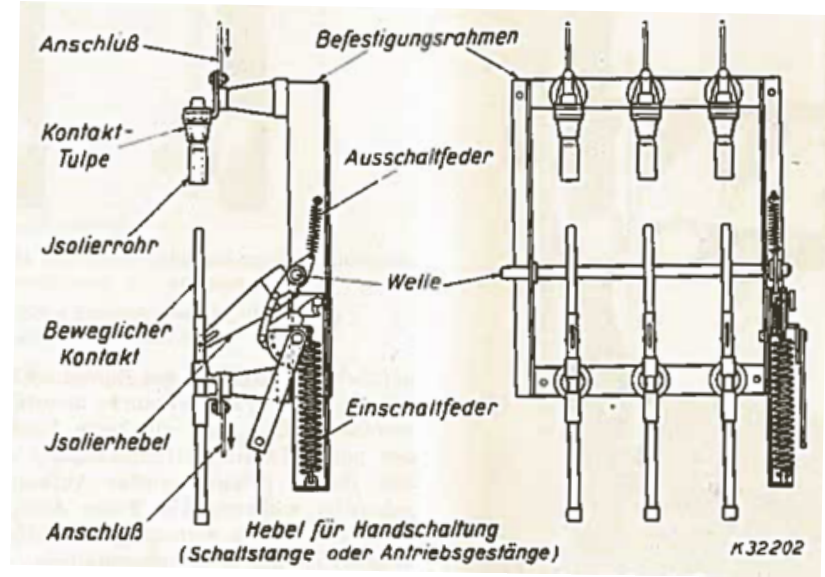

Figure 1 - A $10 \mathrm{kV}$ / 350 A breaker design as presented in Elektrotechnische Zeitschrift in 1935 [1]. Successful interruption of currents up to $1000 \mathrm{~A}$ is reported in the same paper. 


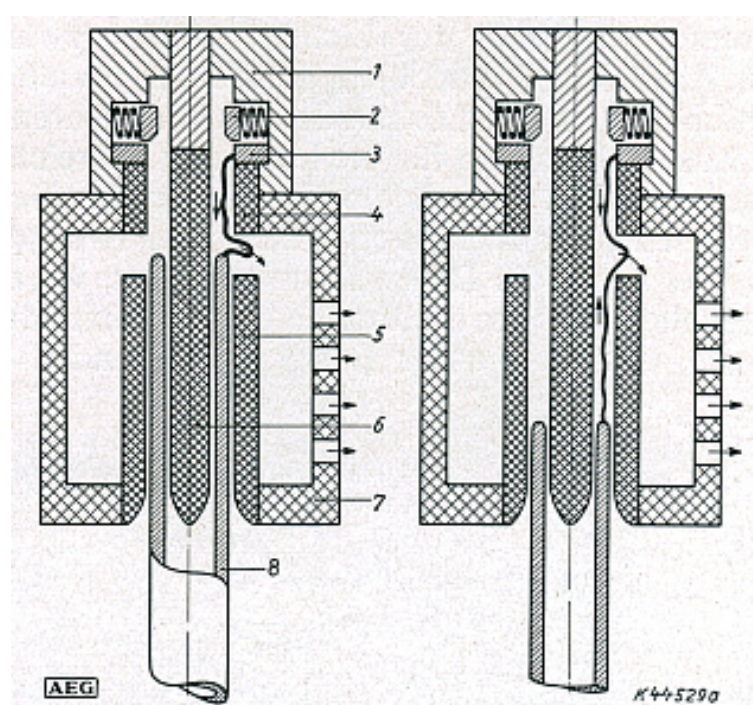

Figure 2 - Example design for a medium voltage switch applying ablation in an annular gap to quench the arc [2].

Some exhaust vents on the outer ablation tube were introduced to limit the wear of ablation material at high currents. At high currents, the ablation is intense already at a short contact distance. As the movable contact passes the vents, a gas flow blows on and quenches the arc. High current arcs do not affect the lower part of the tube, thus saving this part of the tube for the interruption of small currents. When interrupting low currents, the ablation is weaker and a longer part of the tube is needed to reach the necessary level of ablation to quench the arc. In this case, the lower part of the ablation tube is also exposed to the arc. The interruption of high and low currents are illustrated on the left and right-hand side in Figure 2, respectively. In 1957, a refined version of the same breaker design was introduced, but the basic principles were unchanged [3]. Other designs based on the annular-gap ablation design, are presented in [4].

\subsection{Parallel-plate ablation switch}

A popular breaker type has been a parallel-plate ablation design patented in 1943 [5]. A version of this breaker, for ratings up to $36 \mathrm{kV}$ and $1000 \mathrm{~A}$, is still commercially available today [6]. The stationary arcing contact is situated between two ablation plates, with only a few millimeters wide opening in between. When the contacts separate, the electric arc ignites and burns in this narrow gap. The heat of this arc causes an intense ablation of the surface material, and the ablated un-ionized gas blows onto the arc, finally leading to the arc quenching at current zero. A sketch is show in Figure 3.

The movable contact moves out of and away from the ablation plates and stops at a safe clearing distance from the plates. The result is a contact gap that constitutes of two main zones, one ablation dominated zone near the stationary contact and one open-air zone near the movable contact.
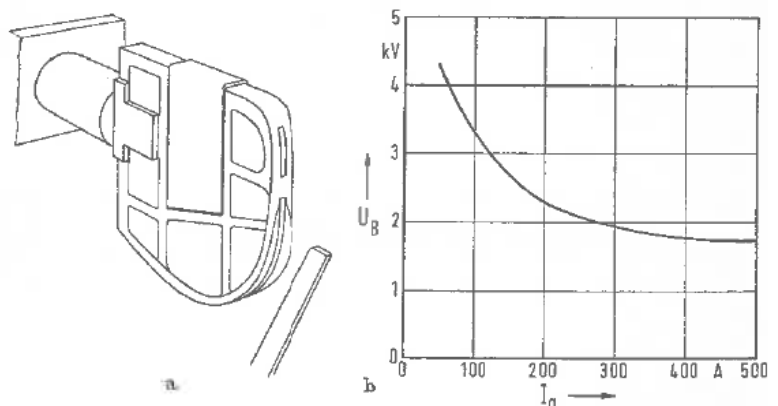

Figure 3 - Parallel-plate ablation switch and its arc voltagecurrent characteristic [4]

\subsection{Combined puffer-ablation switches}

There are some examples of designs that claim to combine ablation with an air blast provided by a puffer arrangement. In a puffer arrangement the movement of one the contact members compresses air that then blows onto the arc. An example of such breaker is depicted in Figure 4. The main quenching mechanism in this breaker is clearly the puffer operation. The ablation element is there mainly to improve arc quenching at high currents [7]. No scientific study has been published on the effect of ablation in such a combined pufferablation switch. It is not clear whether the effect of ablation on the arc quenching is a matter of gas composition in and near the arc or merely a mechanism to increase the gas pressure in the puffer chamber, possibly due to clogging of the nozzle in the high current region.

A more recent design that combines the actions of puffer and ablation is presented by Uchii et al. [8]. The design builds on a well know hybrid self-blast puffer arrangement that exploits the arcs power to heat the gas in the puffer volume, hence increasing the pressure. The novelty is that an ablation element in the puffer volume release gases that increase the pressure even further. The conclusion in [8] is that the ablation element increases the thermal arc quenching capability of the breaker. The effect on dielectric performance is not mentioned. 

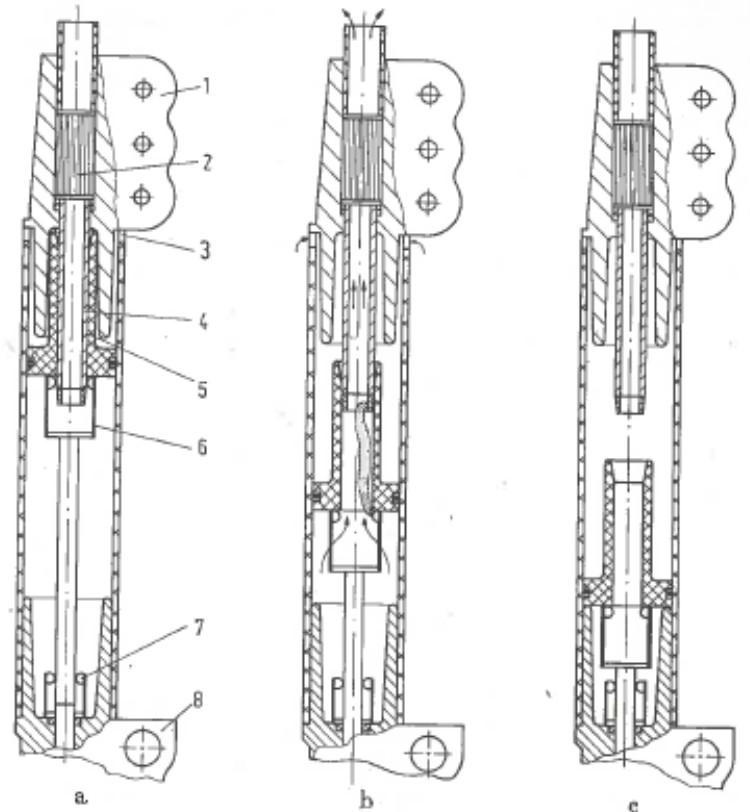

Figure 4 - A combined puffer-ablation switch from BrownBoveri. (a) shows the switch in closed position, (b) the high current region of interruption and (c) the open position. The nozzle (5) is made of a gas-emitting material. The arrows in b illustrate the gas flow as the contact movement compresses the air in the lower chamber [4]

\section{The physics of ablation-assisted switching}

\subsection{Ablation-dominated arcs in the high- current region}

Many publications describe the high-current region of ablation-dominated arcs [9-12]. Most experimental and theoretical studies focus on the arc in a cylindrical tube of ablative material because the dimensions are then reduced to the axial and radial axis. In brief, these arcs are controlled by the axial flow of ablation gasses. Under stable arcing conditions, the surface temperature of the ablation material is at the materials boiling temperature. Between the tube's inner surface and the electric arc plasma, there is a layer of un-ionized ablation vapour. The two zones, the plasma and the vapour, are considered isothermic, with steep temperature gradients in the plasma-vapour interface and the vapour-surface interface. A schematic is shown in Figure 5.

The stationary condition of ablation-dominated arc can be described by the conservation of energy, mass and momentum. The conservation of energy states that the ohmic power dissipated in the arc must be equal to the power transported away. The mass flow of ablation vapour from the tube's inner surface must be equal to the mass flow axially out of the tube opening. Both the added mass and the ohmic heat added from the arc lead to a pressure increase inside the tube, which act as the source of momentum outward from a stagnation point. In the case that both ends of the tube are equally open, the stagnation point will be in the centre of the tube.

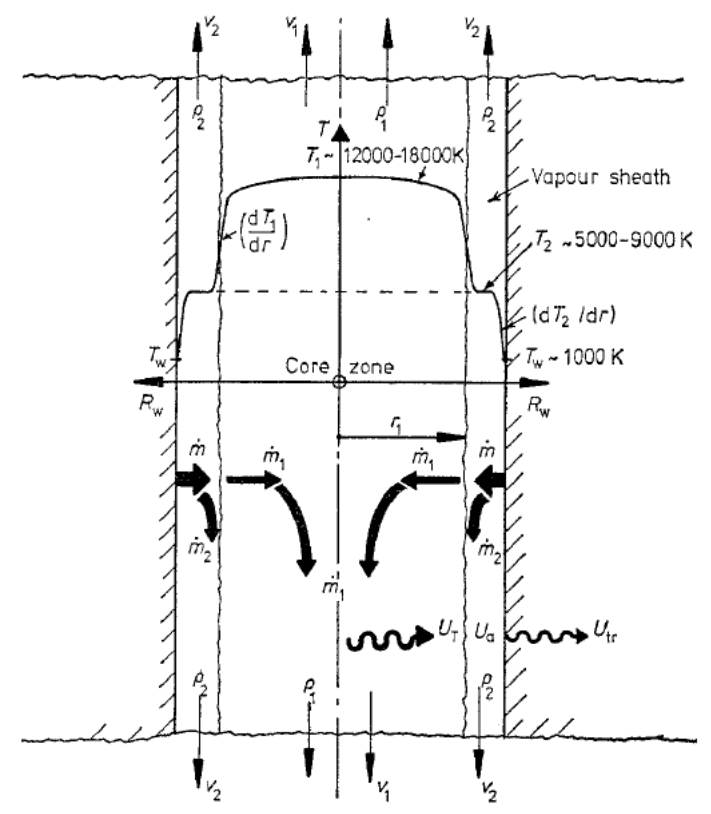

Figure 5 - Fundamental thermo-fluidic parameters in the ablation dominated arc [10].

Establishing the condition of the stable ablationdominated arc can be valuable in understanding such phenomena as nozzle clogging and reversed gas flow in gas blast breakers, as well as arc voltage in current limiting low voltage circuit breaker.

One the other hand, the understanding of the arc in its high current region cannot be directly related the process of arc interruption, but the condition in the high current region provides the starting conditions for the processes around current zero.

\subsection{Ablation-dominated arcs in the current- zero region}

Balestrero et al. [13] present experiments performed on low voltage moulded case circuit breakers (MCCB) and suggest that, rather than the condition in the high current region, the arc characteristics at current zero are predicting the success of the current interruption. The observations made over the last half period of the current (macroscopic evaluators); such as peak arc voltage and total arc energy, show little correlation with the success or failure of the interruption. In contrast, the observations near current zero (microscopic evaluators); such as the current slope at current zero and charge passing through post-arc channel, show a clear correlation with the outcome of interruption.

The processes governing the arc parameters around current zero are extremely fast and difficult to observe. Onchi et al. [14] have performed a theoretical investigation of the effect of polymer ablation on arc quenching properties around current zero. The calculations of change in temperature and electric conductivity were performed with PTFE, POM and a range of fictitious polymers in a cylindrical tube. The conclusion is that the characteristics of the solid 
material, such as evaporation temperature and energy, are of little relevance to the important changes around current zero. The important parameters are the thermal and electric conductivities and mass density, all as functions of temperature. POM shows a significantly faster reduction in temperature and electric conductivity than PTFE.

The experimental approach is important in the research on arc interruption. The process of current interruption is very complex and stochastic of nature and thus hard to predict with confidence. Jonsson et al. [15] has performed experiments comparing the arc quenching capability of different polymers. They confirm that ablated PTFE is a poor arc quenching material compared to other polymers. The general conclusion is that a high content of hydrogen in the ablation gas is beneficial for arc quenching.

\subsection{The dielectric recovery in ablation gasses}

Shea [16] performs a thorough investigation into the mechanisms leading to dielectric failure after interruption and presents experiments applying some of the most common ablation materials in MCCBs, different kinds of fish paper (vulcanized cellulose), Nylon 6/6 and polyester with different fillers. A general observation is that materials producing high pressure at the time of breakdown, show higher withstand voltages. Nylon 6/6 is an exception to this general observation, with high withstand voltage despite of a low pressure relative to other poorer performers. Shea suggests that thermionic emission from the cathode is influencing the withstand voltage. Thermionic emission increases with contact temperature and carbon deposits from the ablation material on the contacts. Carbon and wolfram, a common contact material, are good thermionic emitters. Nylon 6/6 gives a small amount of solid carbon residue, which explains the high withstand voltage observed.

The influence of nozzle ablation on the dielectric properties after current zero is an important topic in high voltage circuit breaker design, even though ablation is not used actively for arc quenching in these breakers. Seeger et al. [17] and Kurz et al. [18] argue that, in a gas blast circuit breaker, the ablation gas inside the nozzle significantly inhibits the dielectric recovery. Initially, they observe a fast increase in dielectric strength for some $100 \mu$ s after current zero, after that the withstand strength stay at a stable level for about $1 \mathrm{~ms}$ before it starts to increase again. During the period of stable withstand voltage, the breakdown happen on or near the inner surface of the PTFE nozzle.

Published investigations into ablation-assisted interruption at ratings relevant for medium voltage switchgear are scarce. Gjendal et al. [19] has published experimental results on a parallel-plate breaker configuration at $6.9 \mathrm{kV}$. They show a remarkable thermal interruption capability, but dielectric recovery is to slow for most voltage characteristics.

In [20] the experimental set-up is slightly improved by closing the arc chamber in all but one direction and splitting the arc column in two by a PTFE wall with a hole for the contact pin. In that way, only one part of the arc column is controlled by ablation, the other part is free-burning in air. The experiments show that the peak value of the recovery voltage is the most important factor for the outcome of the interruption, and that the rate of rise of recovery voltage is of less importance. This makes sense when considering the findings by Seeger et al. [17] and Kurz et al. [18] that the withstand voltage increases rapidly to a certain level, then stays at that level for some time before rising again. In [20], no interruptions were successful when the source voltage was raised to $13,9 \mathrm{kV}$.

\section{Prospects for ablation-assisted current interruption at MV}

Section 3 and 4 of this paper show that ablation-assisted current interruption is an efficient and well-establish method for current interruption, which can also be applied in medium voltage switchgear. On the other hand, it is clear that, although arc-quenching capabilities are very good, there are challenges regarding the dielectric recovery in the presence of ablation material. Extensive development of ablation-based switchgear technology took place in the decades before $\mathrm{SF}_{6}$ was introduced. Despite this effort, most switchgear is rated no higher than $10-20 \mathrm{kV}$. This could indicate that there is a limit for these simple designs around that voltage, and that some fundamentally new design principles are needed to improve voltage ratings further.

The dielectric recovery is obviously a challenge in ablation-assisted switching at medium voltage, but very few investigations on the topic are publicly available. Better understanding of the processes leading to dielectric re-ignition is probably valuable to the development of new compact switchgear designs based on the principle of ablation-assisted switching.

\section{Conclusion}

The increased pressure to remove $\mathrm{SF}_{6}$ from electrical equipment wherever possible has revived the need for research and development in medium voltage switchgear. One of the technologies that could facilitate the transition away of $\mathrm{SF}_{6}$ is the application of ablation for arc quenching. This paper has presented some of the products that were available in the decades before $\mathrm{SF}_{6}$ entered the market and that illustrate the principle of ablation-assisted current interruption.

Not all physical processes of ablation-assisted switching are yet fully understood, but are in part explained through investigations on low voltage moulded case circuit breakers and on nozzle ablation in high voltage switchgear. The medium voltage experiments that have been published indicate that the main challenge in order to exploit ablation-assisted current interruption is the 
slow dielectric recovery in the presence of ablation materials.

On the other hand, over the last decades, not much effort has been put into developing this type of products. With a revived effort, applying new knowledge and new tools, the former switchgear designs could constitute a starting point or source of inspiration for a new generation of $\mathrm{SF}_{6}$-free switchgear.

\section{Acknowledgement}

I want to thank my supervisor at Norwegian University of Technology and Science, as well as Magne Runde, Erik Jonsson and Nina Støa-Aanensen at SINTEF Energy, for valuable feedback and guiding.

This work is part of a project supported by the Research council of Norway.

\section{References}

[1] Mayr, O., Ein neuer Leistungstrennschalter. ETZ, 1935. 56(44): p. 1189-1192.

[2] Petermichl, F., Hartgasschalter. AEG Mitteilungen, 1938(Heft 11): p. 521-523.

[3] Keuneke, W., Hartgasschalter. AEG Mitteilungen, 1957. 47(7/8): p. 285-288.

[4] Erk, A. and M. Schmelzle, Grundlagen der Schaltgerätetechnik: Kontaktglieder und Löscheinrichtungen elektrischer Schaltgeräte der Energietechnik. 1974: Springer-Verlag.

[5] Rawlins, H.L., Load break disconnect. 1943, Google Patents.

[6] 3CJ2 Switch-Disconnectors, in Medium-Voltage Equipment, Siemens, Editor. 2008, Siemens.

[7] Czylok, J.G. and A. Nesse, MittelspannungsSchaltanlagen mit Lasttrennschaltern in Kompaktbauweise. Brown Boveri Mitteilungen, 1967. 54(12): p. 801-808.

[8] Uchii, T., T. Shinkai, and K. Suzuki. Thermal interruption capability of carbon dioxide in a puffer-type circuit breaker utilizing polymer ablation. in Transmission and Distribution Conference and Exhibition 2002: Asia Pacific. IEEE/PES. 2002. IEEE.

[9] Niemeyer, L., Evaporation Dominated High Current Arcs in Narrow Channels. Power Apparatus and Systems, IEEE Transactions on, 1978. PAS-97(3): p. 950-958.

[10] Ibrahim, E.Z., The ablation dominated polymethylmethacrylate arc. Journal of Physics D: Applied Physics, 1980. 13(11): p. 2045.

[11] Ruchti, C.B. and L. Niemeyer, Ablation Controlled Arcs. Plasma Science, IEEE Transactions on, 1986. 14(4): p. 423-434.

[12] Gonzalez, D., H. Pursch, and F. Berger, Experimental investigation of the interaction of interrupting arcs and gassing polymer walls. 2011 Ieee 57th Holm Conference on Electrical Contacts, 2011.
[13] Balestrero, A., et al., Current interruption in lowvoltage circuit breakers. Power Delivery, IEEE Transactions on, 2010. 25(1): p. 206-211.

[14] Onchi, T., Y. Tanaka, and Y. Uesugi, Effect of polymer ablation gas on arc quenching properties around current zero. Electrical Engineering in Japan, 2012. 180(3): p. 32-45.

[15] Jonsson, E., et al., Comparative Study of ArcQuenching Capabilities of Different Ablation Materials. IEEE Transactions on Power Delivery, 2013. 28(4): p. 2065-2070.

[16] Shea, J., Gassing arc chamber wall material effect on post current-zero recovery voltage breakdown, in Electrical Contacts, 2002. Proceedings of the Forty-Eighth IEEE Holm Conference on. 2002. p. 70-79.

[17] Seeger, M., et al., Investigation of the dielectric recovery in synthetic air in a high voltage circuit breaker. Journal of Physics D: Applied Physics, 2005. 38(11): p. 1795.

[18] Kurz, A., et al. Modeling of the dielectric recovery of hot air in insulating nozzles. in Proceedings of the 2012 IEEE International Power Modulator and High Voltage Conference. 2012.

[19] Gjendal, G.J., E. Jonsson, and M. Runde. AblationAssisted Current Interruption in a Medium Voltage Load Break Switch. in ICEC 2014; The 27th International Conference on Electrical Contacts; Proceedings of. 2014. Dresden, Germany.

[20] Gjendal, G.J., Ablation-Assisted Current Interruption in a Medium Voltage Load Break Switch: An Experimental Study with Polypropylene as Ablation Material. 2014. 